\title{
Electro-Hydraulic System Monitoring and Control Using Multivariate Latent Methods
}

\author{
Waleed M.Taha1, Wessam Hussein ${ }^{2}$, Abdulaziz Morgan ${ }^{3}$ \\ ${ }^{1}$ Egyptian armed forces \\ ${ }^{2}$ Egyptian Academy for Engineering and advanced Technology \\ ${ }^{3}$ Ain Shams University \\ Mechanical Power Department, Faculty of Engineering \\ Ain Shams University \\ Cairo, Egypt
}

\begin{abstract}
With the rapid development of technology of hydraulic automation systems is observed in our current era, an ongoing effort to introduce engineering technology, automated solutions in everyday life with an emphasis on the field of hydraulic automation systems. The systems sector develops increasingly their fields of application, whether simple or hydraulic circuits for modern hydraulic automation circuits. This paper outlines the use of a statistical multivariate technique called Principal Component Analysis (PCA) and applies it to the monitoring and control of electro-hydraulic servo systems. The experiments were conducted using on-off and PID approaches to control the position of a hydraulic cylinder under different speeds and different PID coefficients. This approach was implemented using data from position sensors with programmable logic controllers (PLC) with the aid of supervisory control and data acquisition system (SCADA) to control the system and make online monitoring for the behavior of the hydraulic cylinder. The SCADA concept was developed to be a universal means of remote access to a variety of local control modules, which could be from different manufacturers and allow access through standard automation protocols. in practice, large SCADA systems have grown to become very similar to distributed control systems in function while using multiple means of interfacing with the plant. They can control large-scale processes that can include multiple sites, and work over large distances as well as small distances. It is one of the most commonly used types of industrial control systems, both large and small systems can be built using the SCADA concept, these systems can range from just tens to thousands of control loops depending on the application. PCA was used to detect the best speed and coefficient of the process. The proposed technique can be used for electro-hydraulic servo system monitoring in a real-time environment.
\end{abstract}

Keywords: Programmable logic control, Pulse width modulation, Supervisory Control and Data Acquisition, Principal Component Analysis.

\section{INTRODUCTION}

Electro-hydraulic control valves are key hydraulic components for industrial applications and aerospace, which controls electrohydraulic motion. With the development of automation, digital technology, and communication technology, control valves are becoming more digital, integrated, and intelligent in order to meet the requirements of industry[1].

By hydraulic system we mean that a device used to transmit motion and power transmission from the motor to the driven machine. The analysis, design and modeling hydraulic automation systems, based on principles and laws, is widely applicable in fields such as the automotive industry and in robotics $[2,3]$.

SCADA is the most modern tool used for collecting data designed to assist operators in the operation of the any system using realtime and historical information [4]. SCADA system enables human interfaces for controlling and monitoring the physical values of the pneumatic system process and parameters and provide away for different software package to access values from control devices (PLC) which helps to monitor the performance of the pneumatic equipment [5]. 
Also there is an approach for process monitoring using a multivariate statistical technique, namely kernel principal component analysis is studied on an electro-pneumatic valve actuator [6]. Most monitoring techniques presently available describe the variation of one or a limited number of machine components using a heuristic approach which gives limited information about machine condition. Among these techniques, artificial neural network ANNs and its combination with other methods have been the most predominant to date [7-8]. ANNs techniques are characterized as black box approaches which model the relation between different variables to a desired response without giving any information about what happens inside the process. Also some of these approaches are susceptible to missing data due to sensor reliability issues, and some applications use many charts to monitor individual process. In addition, issues associated with collinearity and dimensionality needs to still be specifically addressed in these techniques. Looking for a generic approach to solve many of these limitations can be addressed through the use of multivariate statistical analysis, a data driven modeling approach [9]. Multivariate statistical process control (MSPC) takes a different approach as compared to the other methods mentioned previously; the most fundamental difference with this approach is that the model is based on non-causal empirical correlations extracted from normal plant operating data when only common cause variation exists [10]. The simplicity of this approach is that there is no need for a fundamental model of the system and only data from normal operation needs to be used, which is generally available in some form at most machines. Among the approaches used in multivariate analysis are: a projection method which called (PCA) [11,12] and projection to latent structure (PLS) [13]. Many applications of these two techniques have been successfully applied in other fields of process monitoring [7, 14-19] ranging from batch to continuous processes. (PCA) is the most widely used data-driven technique for process monitoring which has been heavily studied and applied to industrial systems over the past decade. PCA is an optimal dimensionality reduction technique in terms of capturing the variance of the data, and it accounts for correlations among variables. The lower-dimensional representations of the data produced by PCA can improve the proficiency of detecting and diagnosing faults using multivariate statistics. The principal components span a low dimensional subspace used for analysis. The details of linear PCA can be found elsewhere [20]. This paper outlines the use of multivariate projection approach to develop a framework to monitor an electro-hydraulic system under study. By collecting data from displacement transducers under different cylinder speeds. Model based on PCA can be developed into a single user interface at which, system performance can be monitored and controlled using the latent space of these models.

\section{MULTIVARIATE PCA BASIC THEORY}

PCA decomposes the variance and covariance structure of a data matrix by defining linear combinations of the columns in the original matrix. Moreover, PCA extracts information from data sets by computing a smaller data set and other summary information that adequately captures most of the underlying features from the larger data set. The point that needs to be stressed is that the data can be reduced to a size which is more manageable but contains the features that are often of interest. PCA extracts a score matrix, T, and a loading matrix, $\mathrm{P}$, from $\mathrm{X}$. These matrices have the following dimensions:

$$
\mathrm{X}: \mathrm{N} \times \mathrm{K} \quad \mathrm{T}: \mathrm{N} \times \mathrm{A} \quad \mathrm{P}: \mathrm{K} \times \mathrm{A}
$$

We call the first column of $\mathrm{T}$ and $\mathrm{P}$ by their shorter forms, $\mathrm{t}_{1}$ and $\mathrm{p}_{1}$ respectively. The lower case letters indicate that these are vectors, upper case letters indicate matrices. Extracting only one principal component (that is, a single score vector and loading vector) gives:

$\mathrm{X}=\mathrm{t}_{1} p_{1}^{T}+\mathrm{E} 1 \quad$ and extracting a second principal component: $\mathrm{X}=\mathrm{t}_{1} p_{1}^{T}+\mathrm{t}_{2} p_{2}^{T}+\mathrm{E} 2$

We continue in this manner until we extract principal components and then group these score and loading vectors to form matrices T and P:

$$
\mathrm{T}=[\mathrm{t} 1 \mathrm{t} 2 \ldots \mathrm{tA}] \text { and } \mathrm{P}=[\mathrm{p} 1 \mathrm{p} 2 \ldots \mathrm{pA}]
$$

The eigenvectors of the real symmetric matrix XTX give us exactly the loading matrix P. This is the loading matrix obtained by extracting all principal components. Once we have $\mathrm{P}$ we use the last line of the following relationship to compute $\mathrm{T}$ :

$$
\mathrm{TPT}=\mathrm{X} \quad \mathrm{TPTP}=\mathrm{XP} \quad \mathrm{T}=\mathrm{XP}
$$

A more in-depth discussion, which also highlights some geometric concepts of PCA, can be found in [13]. The algorithm used to calculate the PCA is the NIPALS (Nonlinear Iterative Partial Least Squares) algorithm [12,21].

\section{EXPERIMENTAL SETUP}

The position control for the hydraulic system consists of the control circuit, hydraulic system and the potentiometer (position sensor). The system has been installed on the platform; the position sensor is monitoring the piston of the hydraulic cylinder and sends a signal to the electronic circuit. Used the proportional directional control valve is consist of two solenoids to controlling the spool valve based on the varying voltage signal coming into the controller, which are applied to the solenoids, change the position 
of a spool valve, this will cause a change in the flow rate of the oil that goes past into the cylinder Causing to creating a varied in the position of the hydraulic cylinder rod. Thus the controller will detect the error between the desired position and measurement position of a hydraulic cylinder rod. The position sensor sends a digital/analog signal to the controller unit and telling the controller justly where the hydraulic cylinder rod is through its stroke. The control unit will calculate the error of position and it will send a voltage signal to the proportional directional control valve to control the flow rate of oil in the cylinder to move the cylinder rod to extend or retract the cylinder rod to control the certain position.

Fig. 1 shows a schematic diagram of the experimental test rig while Fig. 2 shows a photograph for the real rig. It consists of a double acting cylinder of $38 \mathrm{~mm}$ core and $200 \mathrm{~mm}$ stroke controlled by a proportional 4/3 directional control valve operates by two solenoids The working fluid is a hydraulic oil whose pressure is controlled by a pressure control valve. The cylinder rod displacement is measured by a linear position sensor. A proportional 4/3 directional control valve with two solenoids is used to control the cylinder motion. The solenoids operate by a PWM electric signal according to the controller command. The control circuit consist of a PLC (WECON LX2E) connect with computer by USB to Serial converter .SCADA software is used to acquire data of the cylinder piston position, then makes the necessary calculation for the controller and finally provides the PWM circuit with the necessary voltage value to drive the valve solenoids to control the oil mass flow rates coming into and out of the cylinder in order to control the piston position as required.

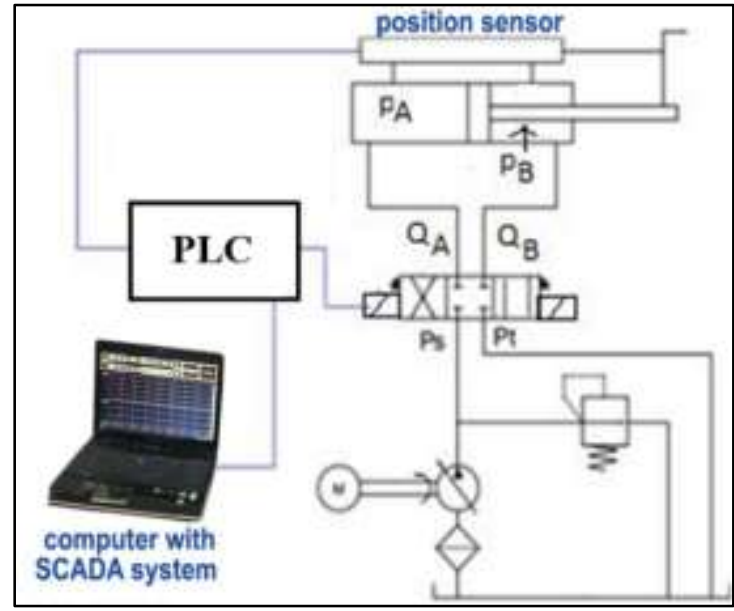

Figure 1: Schematic diagram of electrohydraulic system

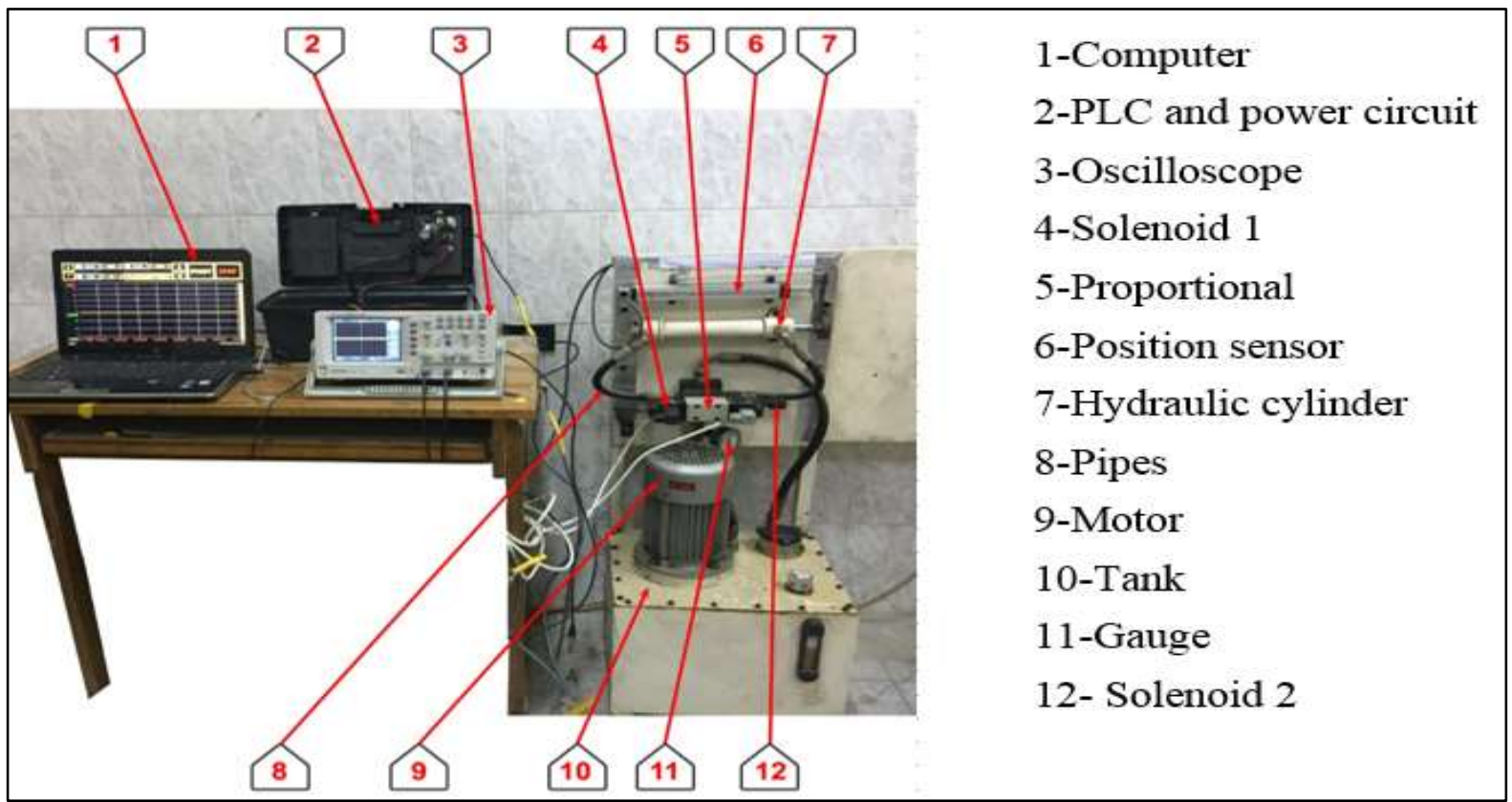

Figure 2: Photograph of Test Rig 


\section{CONTROL DESIGN}

The controller is a part of the feedback control system, shown in Fig. 3, which controls the position of the valve spool so that the position of the piston cylinder is precisely tracked according to the input reference.

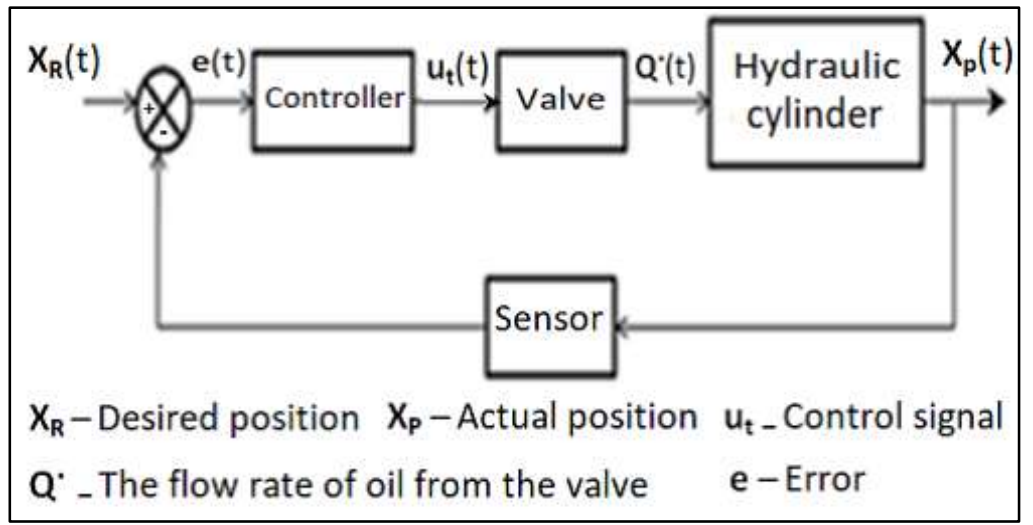

Figure 3: The block diagram of hydraulic system with feedback control

The principle of PID control algorithm applied in position closed loop control system is shown below:

$u(t)=k_{p} e(t)+k_{i} \int_{0}^{t} e(t) d t+k_{d} \frac{d e(t)}{d t}$

Integrated PID closed-loop configuration panel was used in PLC software to construct a closed loop programming as shown in Fig. 4. Ideal control effect can be achieved by adjusting the parameters of the closed loop program such as,

$\mathrm{Kp}, \mathrm{Ki}$ and $\mathrm{Kd}$.

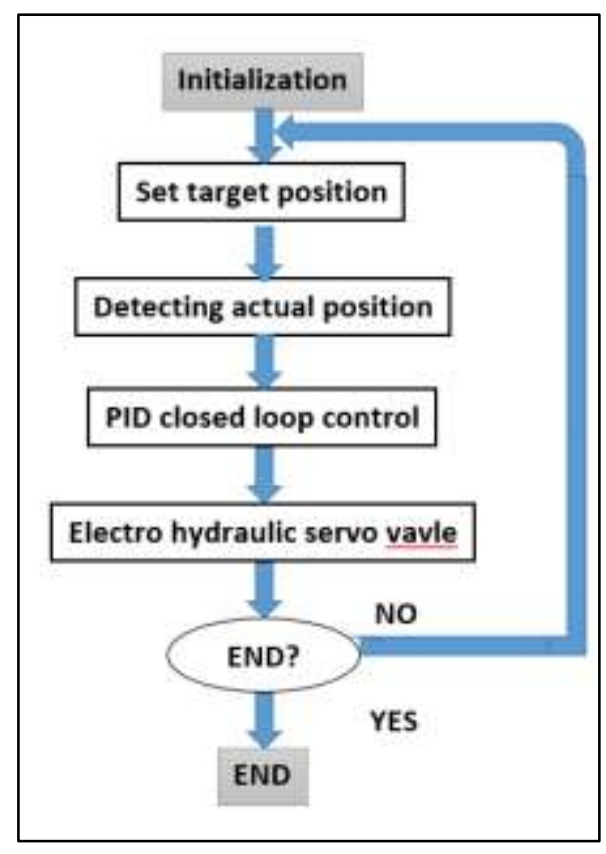

Figure 4: Position closed loop control

\section{MODEL BUILDING, CONFIGURATION AND RESULTS}

The multivariate PCA model is a linear model given in matrix form by: $X=\mathrm{TP}^{\mathrm{T}}+\mathrm{E}_{\mathrm{A}}[9,18]$. Four variables were used to generate the input matrix " $X$ ". These variables represent the reference start and target points in addition to the actual values of the time domain records of the sensory signals (actuator displacement) using both on-off and PID control respectively. All of the models were built using SIMCA-P code developed by Umetrics based on PCA [6]. The PCA model is based on building classes or clusters using existing information inside the data (unsupervised learning). The model is established using a set of experimental runs called the training set that represents the normal operation of the system. Finally, regression is made on the matrix X based on NIPALS algorithm. 
To explore the collected data a first model using PCA was built using all the dataset by cross-validation, now obtaining a two component model, with: R2X(cum): 0.65, Q2(cum) : -0.16. It is seen that the model factor Q2cum, is very small, but it is still better to first get a general look at the whole dataset without removing any variable or observation. Figure 5 illustrates a scatter plot of the two score vectors ( $\mathrm{t} 1$ and $\mathrm{t} 2$ ) of the model using a set of 490 observations. It provides a clear vision of the dispersion of the data, showing different groups of data being discriminated along the first two components. These groups is separated according to the different speeds (50\% - 75\% -100\% of the total speed) and control modes (On-Off and PID). One can see that PID data shows better performance more than On-Off mode and this can be seen by examining the loading plot figure (6).

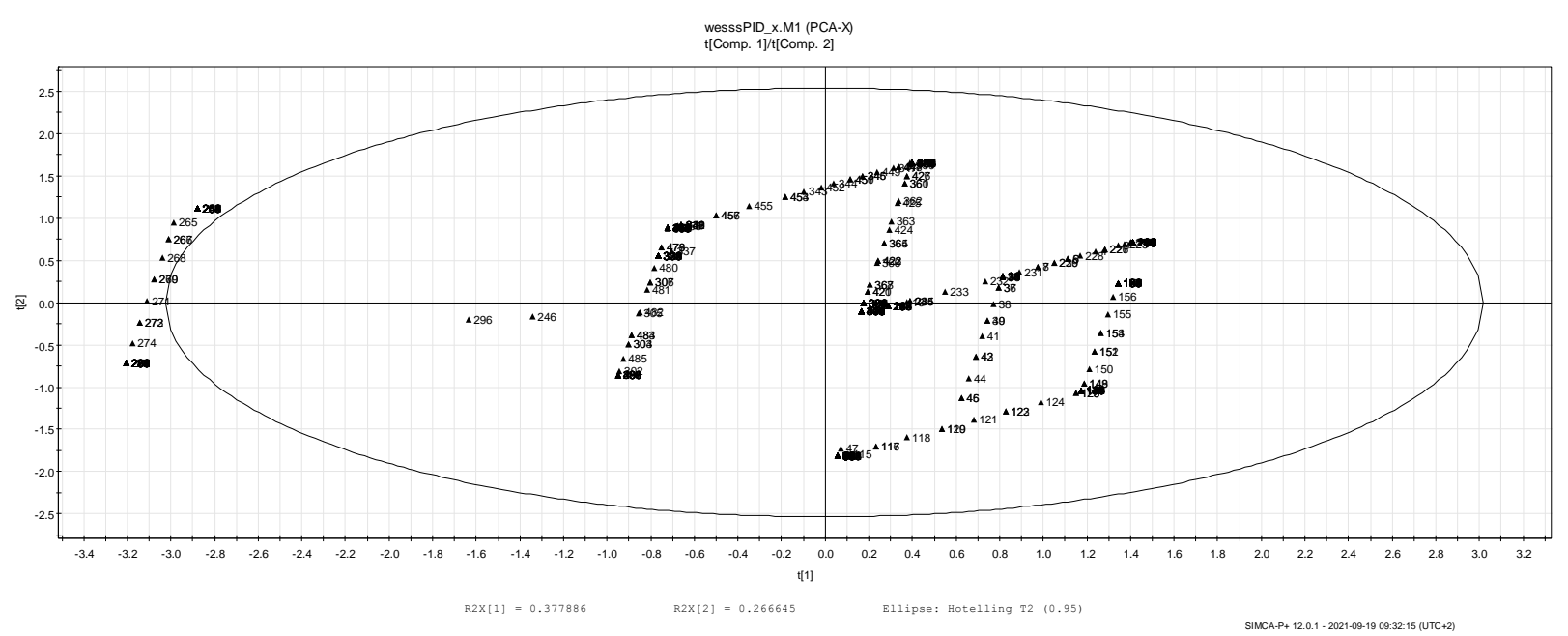

Figure. 5: A scatter plot of the two score vectors (t1 and t2) of the PCA model

The loading plot clarify the relation between the 4 variables. One can detect that PID mode is close to the start and target reference points over the On-Off mode. In addition, the score and loading plots are superimposed; this means that variables lying in each quarter of the loading plot are contributing to the changes between the observations in the score plot.

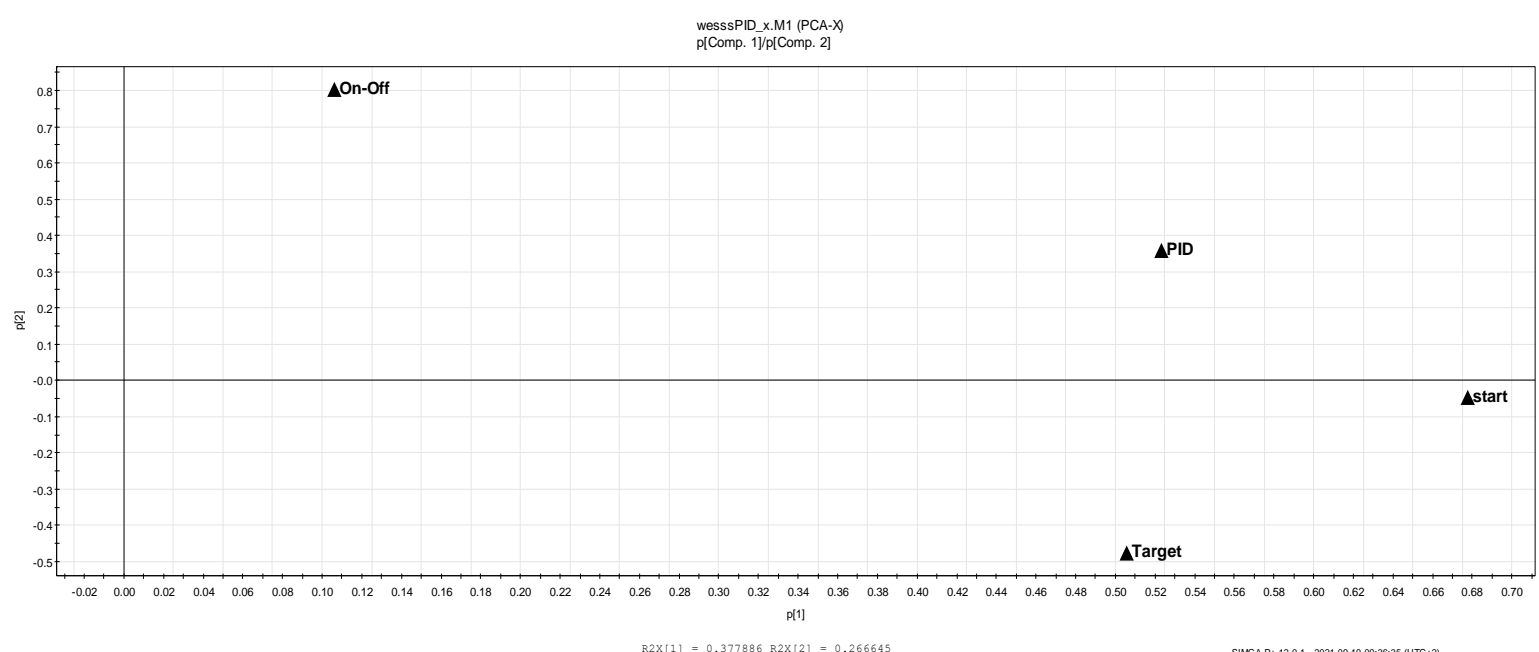

Figure. 6: PCA-X loading plot of four variable PID, On-Off and the two targets.

A set of nine trials was made to adjust the PID coefficients during online operations as shown in table 1. 
Table 1: Different values for PID coefficients

\begin{tabular}{|c|c|c|c|c|c|c|c|}
\hline No. & $\mathbf{K p}$ & $\mathbf{K i}$ & $\mathbf{K d}$ & No. & $\mathbf{K p}$ & Ki & Kd \\
\hline $\mathbf{1}$ & 700 & 5 & 1 & $\mathbf{6}$ & 725 & 5 & 1 \\
\hline $\mathbf{2}$ & 800 & 0 & 0 & $\mathbf{7}$ & 800 & 5 & 2 \\
\hline $\mathbf{3}$ & 700 & 5 & 0 & $\mathbf{8}$ & 800 & 5 & 1 \\
\hline $\mathbf{4}$ & 650 & 5 & 1 & $\mathbf{9}$ & 700 & 5 & 2 \\
\hline $\mathbf{5}$ & 725 & 5 & 2 & & & & \\
\hline
\end{tabular}

A new model was built using PID data during the nine trials, now obtaining a two component model, with: R2X(cum): 0.60, Q2(cum) : 0.3. Figure (7) shows the loading plot for the nine experiments, One can notice that trial 8 has the lower variance over other trials and this is clarified in a bar plot which summarizes the results as shown in figure (8).

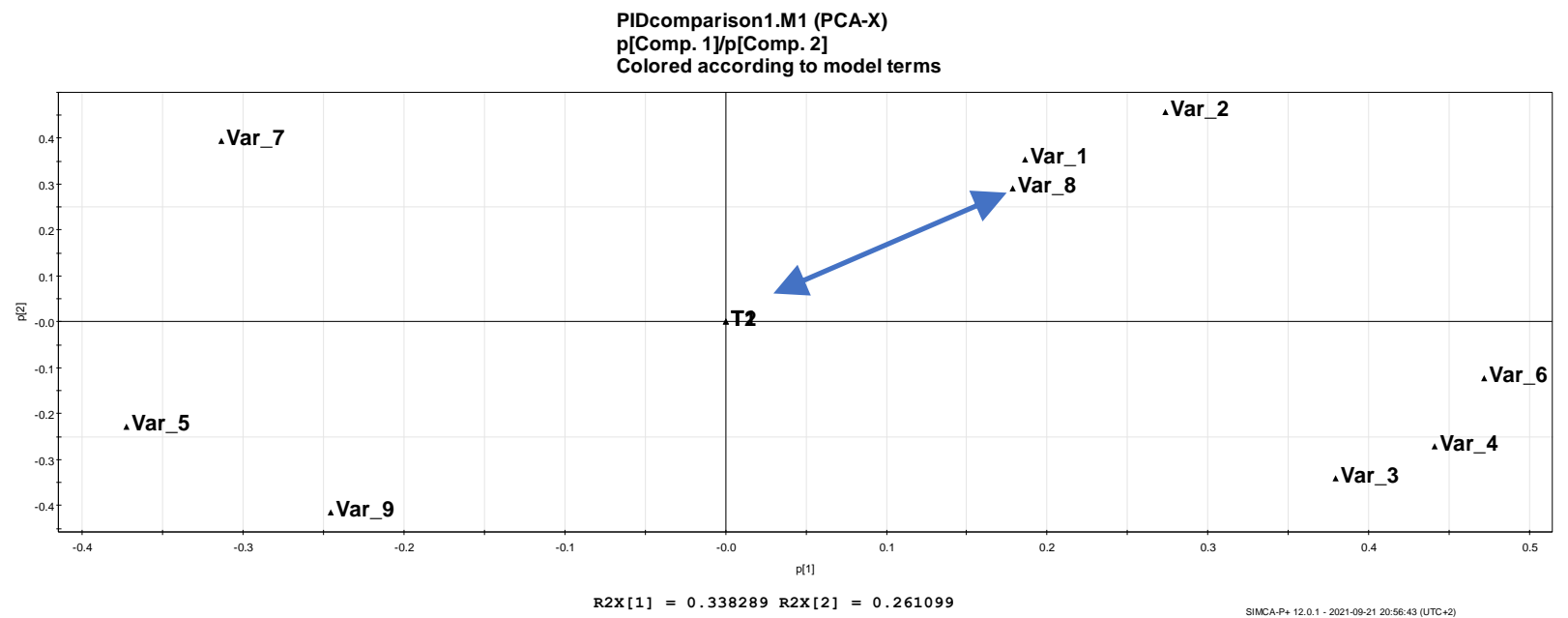

Figure.7: PCA-X loading plot of nine PID trials.

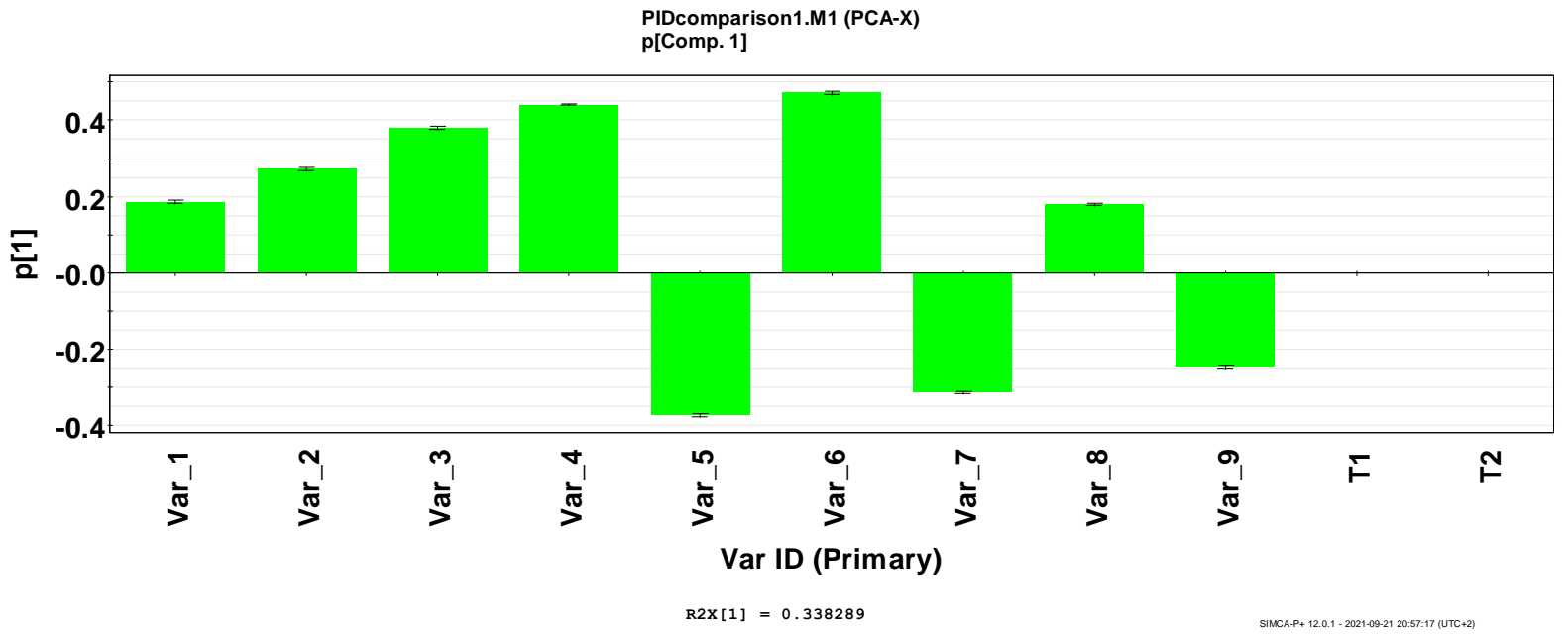

Figure. 8: PCA-X loading bar plot of nine PID trials.

More investigation carried out using the time series plot of a new model. The new model was built using 2355 observations of the dataset, now obtaining a two component model, with: R2X(cum): 0.60, Q2(cum) : 0.30. Figure 9 illustrates the time series plot of 
the four variables under study: On-Off, PID and the two reference targets. The data includes three different zones according to PID different coefficients.

One can notice that both On-Off and PID mode (blue line) shows a high overshoot with $\mathrm{Kp}=900, \mathrm{Ki}=9$ and $\mathrm{Kd}=5$ in the third zone (1570 - 2355). Another trial to change the coefficient shows also a better performance with lower overshoot with $\mathrm{Kp}=700$, $\mathrm{Ki}=9$ and $\mathrm{Kd}=5$ in the second zone (785-1570). A final trial gives modified $\mathrm{Kp}=700, \quad \mathrm{Ki}=5$ and $\mathrm{Kd}=1$ which results in a clear reduction of the overshoot of the PID mode over On-Off mode.

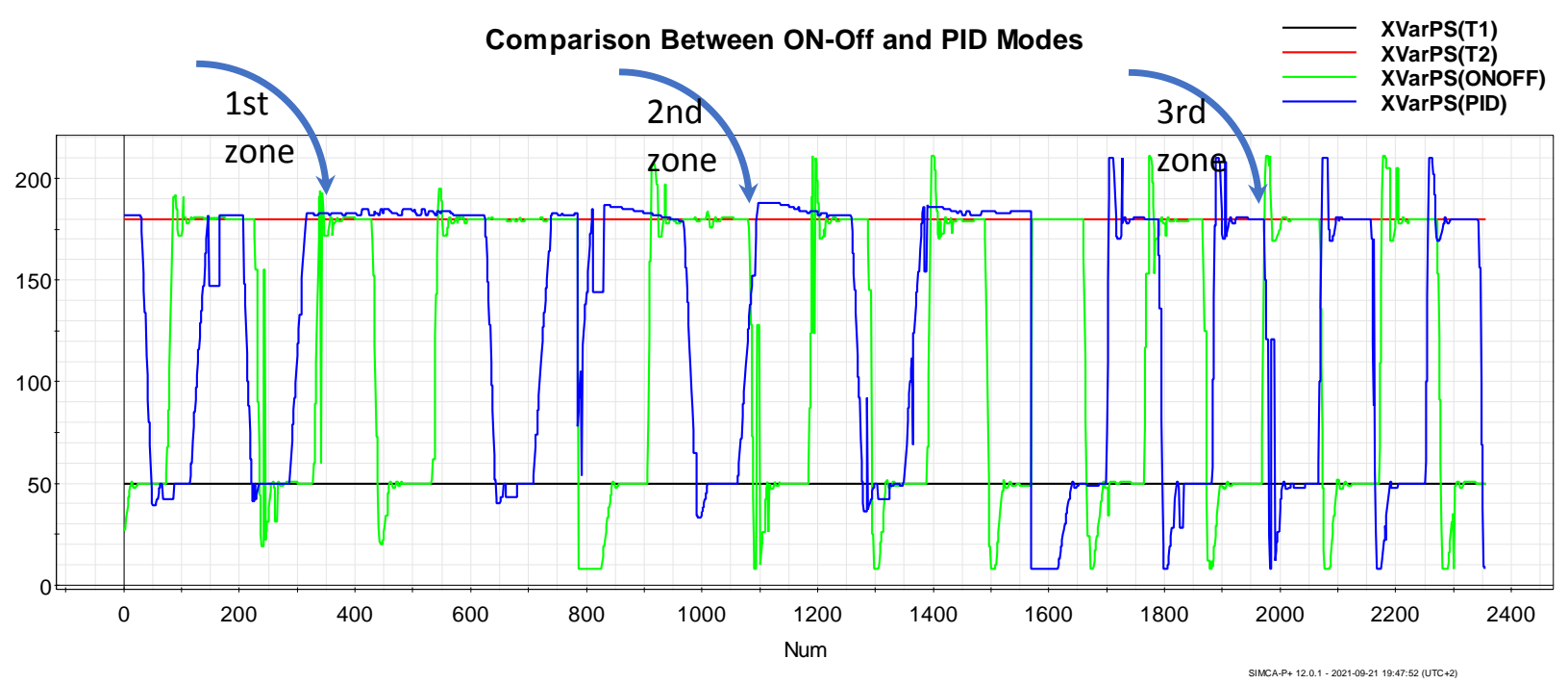

Figure. 9: Time series of actual values using ON-Off and PID Modes for different conditions

\section{CONCLUSION}

The application of multivariate techniques to monitor an electro hydraulic system is the main contributions to the development of a monitoring system. The experiments were conducted using on-off and PID approaches to control the position of a hydraulic cylinder under different speeds. This approach was implemented using data from position sensor with programmable logic controller (PLC) with the aid of a supervisory control and data acquisition system (SCADA) to control the system. PCA was used to detect the best speed and inadequacies of the process. The results shows the superiority of PID control over on-off control. Moreover, SCADA approach gives the advantage to adjust PID parameters online to save more time. The proposed technique can be used for electro- hydraulic servo system monitoring in a real-time environment.

\section{REFERENCES}

[1] Bing Xu, Jun Shen, Shihao Liu, Qi Su and Junhui Zhang "Research and Development of Electro-hydraulic Control Valves Oriented to Industry", Chinese Journal of Mechanical Engineering, 2020.

[2] Hydraulic automatic control systems, N. Pantazis, Publisher ION .

[3] Hydraulic Control Systems, Herbert E. Merritt.

[4] Stouffer, Keith, and Joe Falco. "Guide to supervisory control and data acquisition (SCADA) and industrial control systems Security." Computer Security Division Information Technology Laboratory, MD 20899-893[Accessed Jun, 2018].

[5] Hala, H. H. and Maher, Y. S. "Pneumatic Control System of Automatic Prodution Line Using Two Method of SCADA/HM, Implement PLC", Al-Khwarizmi Engineering Journal, Vol. 15, No. 3, pp. 16-28, 2019.

[6] Sang-Oak Song, Gibaek Lee and En Sup Yoon, "Process monitoring of an electro-pneumatic valve actuator using kernel Principal component analysis", School of Chemical Engineering, Seoul National University, 2002 IFAC

[7] MacGregor J.F., Yu H., Munoz S. and Cerrillo J., "Data-based latent variable methods for process analysis, monitoring and control”, Journal of Computers and Chemical Engineering 29, 1217-1223, 2005.

[8] P. Nomikos and J. MacGregor, "Monitoring batch processes using multiway principal component analysis", 
International Journal of Advances in Scientific Research and Engineering (ijasre), Vol 7 (10), October -2021

AIChE J., Vol.40, 1361, 1994.

[9] Eriksson L., Johansson E., Wold N.K. and Wold S., "Multi- and Megavariate data analysis, principles and applications" Umetrics AB, Sweden, 2006.

[10] Kourti T. and MacGregor J. F., "Multivariate SPC methods for process and product monitoring", Journal of Quality technology, Vol.28, 4, October, 1996.

[11] Wold S., Esbensen K. and Geladi P., "Principal component analysis", Chemometrics and intelligent laboratory Systems, 2: 37-52, 1987.

[12] Jackson J.E., “A User’s Guide to Principal Components”, Wiley, New York, 1991.

[13] Wold S., Sjostrom M. and Eriksson L., "PLS-regression: a basic tool of chemometrics", Chemometric and intelligent laboratory systems, 58:109-130, 2001.

[14] Lennox B., Hiden H.G., Montague G.A., Kornfeld G. and Goulding P.R., "Application of multivariate Statistical process control to batch operation”, J. Computers and chemical engineering, V24, n2, July, PP. 291296, 2000.

[15] Goulding P.R., Lennox B., Sandoz D.J., Smith K.J. and Marjanovic, "Fault detection in continuous processes using multivariate statistical methods", International journal of systems science, 31(11), pp. 1459-1471, 2000.

[16] Yon S., Landry J., Wold N.K., Pepe and Wold S., "Multivariate process monitoring and early fault detection (MSPC) using PCA and PLS”, Plant automation and decision support conference, September 21-24, San Antonio, Texas, 2003.

[17] Chen J. and Liu K.C., "On-line batch process monitoring using dynamic PCA and dynamic PLS models", J. Chemical engineering science 57:63-75, 2002.

[18] Kourti T., "Abnormal situation detection, three-way data and projection methods robust data archiving and modeling for industrial applications", Annual reviews in control 27: 131-139, 2003.

[19] P. Nomikos and J. MacGregor, "Monitoring batch processes using multiway principal component analysis", AIChE J., Vol.40, 1361, 1994.

[20] Jolliffe, I.T., "Principle component analysis", springer virlag, New York, 1986.

[21] Leopold Simar and Wolfgang Hardle, "Applied Multivariate Statistical Analysis", Tech method and data technologies, Springer Verlag, Berlin and Louvain-la-Neuve, 2003.

[22] Li Yanjie, Cui Tianyu and Wang Hai et al. Proportional Valve Controlled Hydraulic Cylinder PID Closed Loop PLC Software [J]. PID Journal of Shen Yang Ligong University, 2013, 32 (4): 37-40. 